\title{
AUTOMATIC TUNING OF A RETINA MODEL FOR A CORTICAL VISUAL NEUROPROSTHESES USING A MULTI-OBJECTIVE OPTIMIZATION GENETIC ALGORITHM
}

\author{
ANTONIO MARTÍNEZ-ÁLVAREZ* \\ Department of Computer Technology, University of Alicante, Carretera San Vicente s/n, Alicante, 03690, Spain \\ E-mail: amartinez@dtic.ua.es \\ www.ua.es \\ RUBÉN CRESPO-CANO \\ Department of Computer Technology, University of Alicante, Carretera San Vicente $s / n$, \\ Alicante, 03690, Spain \\ ARIADNA DÍAZ-TAHOCES \\ Institute of Bioengineering, University Miguel Hernández and CIBER BBN, Av. de la Universidad s/n \\ Elche (Alicante), 03202, Spain \\ SERGIO CUENCA-ASENSI \\ Department of Computer Technology, University of Alicante, Carretera San Vicente s/n, \\ Alicante, 03690, Spain \\ JOSÉ MANUEL FERRÁNDEZ VICENTE \\ Telecomm School, Universidad Politécnica de Cartagena, Campus Muralla del Mar s/n, Cartagena (Murcia), \\ 30202, Spain \\ EDUARDO FERNÁNDEZ JOVER \\ Institute of Bioengineering, University Miguel Hernández and CIBER BBN, Av. de la Universidad s/n \\ Elche (Alicante), 03202, Spain
}

\begin{abstract}
The retina is a very complex neural structure, which contains many different types of neurons interconnected with great precision, enabling sophisticated conditioning and coding of the visual information before it is passed via the optic nerve to higher visual centers. The encoding of visual information is one of the basic questions in visual and computational neuroscience and is also of seminal importance in the field of visual prostheses. In this framework, it is essential to have artificial retina systems able to function in a way as similar as possible to the biological retinas. This paper proposes an automatic evolutionary multi-objective strategy based on the NSGA-II algorithm for tuning retina models. Four metrics were adopted for guiding the algorithm in the search of those parameters that best approximate a synthetic retinal model output with real electrophysiological recordings. Results show that this procedure performs well when different trade-offs has to be considered during the design of customized neuro prostheses.
\end{abstract}

Keywords: Retinal modeling; Visual neuroprostheses; Multi-objective optimization; NSGA-II; Evolutionary search.

*Corresponding author: amartinez@dtic.ua.es 


\section{Introduction}

The retina is a neural circuit composed of different cell classes that capture the light from the external visual world and convey this information to the brain. The retina integrates a rich set of specialized cells and complex neural structures, which are sensitive to color, light intensity, image movements, edges detection, and many other valuable characteristics for the sense of sight. Using these structures and neural circuits, the retina performs chromatic and achromatic spatio-temporal processing of visual information, and finally encodes this information into spike trains that are delivered to higher visual centers via the optic nerve. This process has to be unequivocal and fast, in order to ensure object recognition for any single stimulus presentation within a few hundreds of milliseconds. Therefore the question of how this information about the external world is compressed in the retina, and how this compressed representation is encoded in spike trains is one of the basic questions in visual and computational neuroscience ${ }^{1}$ This knowledge is also of seminal importance for the development of useful cortical prostheses capable of eliciting visual percepts in profoundly blind people through direct stimulation of visual cortex $\sqrt[2[3]{3} \text { Thus one of the }$ major challenges in this approach is the design and development of a retina-like platform able to transform the visual world in front of a blind individual into a set of electrical signals that can be used to stimulate, in real time, the neurons at his/her visual cortex. These signals should be as similar as possible to the output signals of the real retina, and the tasks are comparable to the present challenges faced by the brain-computer interfaces and their inherent technologies. ${ }^{4}$ The full description of this problem

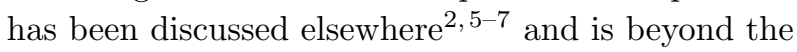
scope of this paper, but Fig 1 summarizes the basic processing blocks of the bioinspired retinal model that we are currently using.

This model is based on electrophysiological recordings from populations of retinal ganglion cells and is able to work in real time. Nevertheless, before proceeding with in-vivo animal experimentation, fine tuning of the involved retinal models is needed. Tuning a retinal model is the process of adjusting the parameters and functions of a retinal model to best match its output with true biological records. Thus, appropriate matching metrics are needed to assess the tuning of our synthetic retinal models.

From a mathematical point of view, the operation of the first two processing blocks from Fig. 1, labeled as Stage 1, can be modeled as a weighted combination of different well-known convolutive spatiotemporal image filters such as Gaussians, Difference of Gaussians (DoG), Laplacian of Gaussian (LoG), Gabor, Sobel, etc (see Stage $_{1}$ on Eq. (1)). This combination results as an activity matrix feeding an Integrate $\&$ Fire model, that calculates the specific spike patterns that can be sent to each single electrode (see Stage $_{2}$ on Eq. (2)). Stage 3 which represents the potential electrode re-mapping is beyond the scope of this paper. Each processing block from the retinal model (stage $1+$ stage 2 ) has many parameters to be tuned. Many of them move in a continuous dynamic range (e.g. $\sigma$ parameters for a Gaussian of Difference of Gaussian filters) and some of them can be modeled as natural numbers (e.g. Kernel size $(N \times N)$ of a convolutive filter). With such an infinite search space to explore, the process of adjusting those parameters represents a difficult problem to be solved.

Several models have been proposed to mimic the retina behavior $8 \sqrt{9}$, leading to different strategies for fitting their parameters. Linear-NonlinearPoisson model (LNP) ${ }^{10}$ generates at any instant in time the linear response by multiplying the stimulus by a temporal weighting function, pointwise, and summing the result. Nonlinearities associated to ganglion cell responses (e.g. spike threshold) are modeled as "static", meaning that the linear response can be passed through an input-output function that is invariant over time. Usual methods to fit the LNP model includes the cross-correlation between the stimulus and the response to compute the linear filter coeficients and the mean squared error (MSE) to fit the parameters of a Gauss cumulative distribution (gcd) that works like non-linear function. Zaghloul et al. 11, proposed also a gcd to model the simultaneous low and high contrast response of Y-type RGCs. Ozuysal et al. ${ }^{12}$ improves the LN response to high contrast variations adding an adaptive mechanism, yielding a system with a linear temporal filter, a static nonlinearity, and an adaptive kinetics block. In any case, gcd parameters are calculated to minimize the MSE between model estimation and spike rate response, limiting the problem to a unique objective optimization process. Anyway, LN model predicts the spike rate but not actual spike times; 


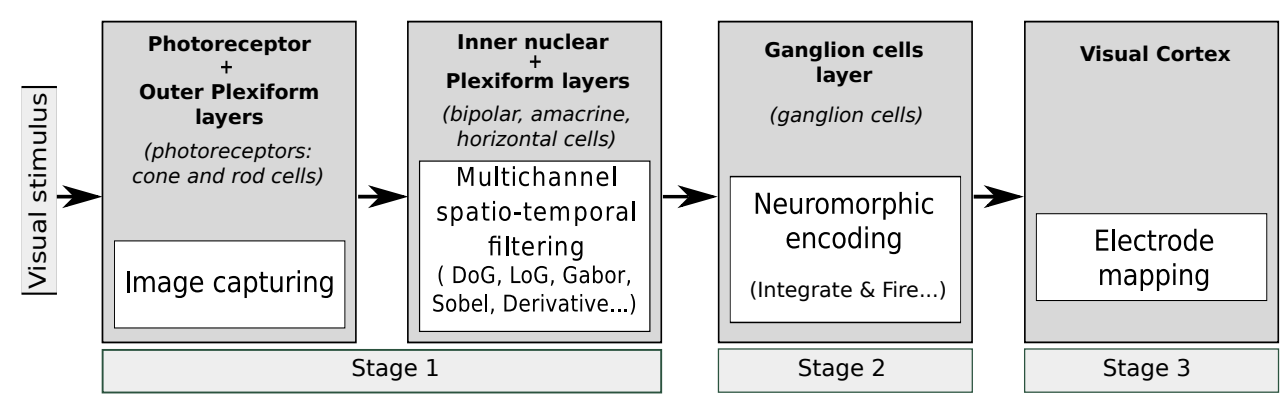

Figure 1. Functional processing blocks of the bioinspired retina model under study.

spiking is modeled as a Poisson process, defined by a rate (with equal mean and variance), but spike times are otherwise random.

On the other hand, integrated-and-fire spike generator (IF) has been reported as a good model to provide a detailed functional description of the light responses of individual retinal ganglion cells (RGCs) of ON and OFF classes 13. Regarding the optimization of the model parameters, Pillow et al. ${ }^{13}$ used a likelihood function to fit an IF model applied to individual EGCs (class ON and OFF). The same method was subsequently applied for optimizing a Generalized Linear-Nonlinear-Poisson Model 14. GLM simultaneously captures both the stimulus dependence and detailed spatio-temporal correlations in EGC population responses. In both cases, IF and GLM, authors maximize the likelihood of the observed responses with a log-concave function which does not contain any local maxima. The variance explained, or its equivalent Poisson correlation coefficient, was used to measure the quality of the response. However, likelihood approach represents an oversimplification of the problem which ensures the model can be fitted reliably and tractably using simple gradient techniques. Spiking neural networks, used to solve other kind of problems 15 17, were also applied to build a generalized integrated-and-fire neuron model ${ }^{18}$ and to describe the response of different nature neural populations 1921 including high level functionalities of the visual system 22 .

Recently the potential of deep networks for learning meaningful features has been demonstrated on a number of visual tasks 23 26. Deep Belief Networks have also been shown suitable for modeling feature detection in the retina ${ }^{27}$ and visual areas V1 \& V2 28. Regarding retina modelling purposes, Turcsany et al. ${ }^{27}$ promote the use of multi-layer deep networks owing to their ability to extract a hierarchy of distinctive features from data and provide the required flexibility for modelling our incomplete knowledge about the roles of cells and circuits of the visual pathway. A four layer network, each one corresponding to a biological layer, was unsupervised trained in two phases; a pre-training phase where by the multi-layer representation is learnt one layer at a time using an Restricted Boltzman Machine on each layer, followed by fine tuning using backpropagation. As usual in deep learning techniques, a large data set is required for fitting the network parameters, so authors carried out simulations using just synthetic images without any biological recordings. Furthermore, other architectural network settings difficult to adjust, as the number of hidden nodes per layer and learning rate values, had to be fitted by repeating the experiment and taking the best performed configurations.

In this context, our approach optimizes the IF model by using four metrics apart of the variance explained or correlation coefficient. Those metrics describe not only the relevant features of the response in terms of spike rate, like the shape of Post-Stimulus Time Histograms (PSTH) and the firing rate, but also improves the prediction of the spike times by measuring the firing response in time for each individual RGC and takes into account the receptive field areas. This way, the traditional fitting of the model parameters, undertaken with simplest approaches, becomes a more complex multi-objective problem. To overcome this problem, we present an extension of our previous work presented in ${ }^{29}$ where a proposal to use an automatic evolutionary multi-objective strategy exploiting the synergies of neural networks and evolutionary computation 30 . This strategy allows to explore the trade-offs among all objectives and 
generate retinal models which better approximate to the real biological recordings. There are other evolutionary approaches to tackle the multi-objective optimization. These methods can be classified into two different groups. The first group is defined with relatively simple algorithms based on Pareto ranking $31 \quad 32$. The second group includes elitist algorithms that emphasize computational efficiency, like Non-dominated Sorting Genetic Algorithms NSGAII ${ }^{33}$ and NSGA-III ${ }^{34}$, Strength Pareto Evolutionary Algoritm 2 (SPEA2) 35, Multi-objective Evolutionary Algorithm based on Decomposition with PBI (MOEA/D+PBI) 36, Multiple Single Objective Pareto Sampling (MSOPS) 37 , or Hypervolume Estimation Algorithm (HypE) 38. Among them, the widely contrasted and well-known NSGA-II strategy was selected for this purpose.

The main contribution of our work is twofold. Firstly, our approach uses a comprehensive description of the retina response, supported by biological evidences and based in the four features previously mentioned. We propose four different metrics which capture each relevant characteristic and allow a more accurate rendering of the biological response. Additionally, in order to rebuild the receptive fields of every unit, our technique uses a novel method of response fusion reported in 39 . Secondly, the proposed strategy exploits the synergies of neural networks and evolutionary computation 40, enabling neuroprostheses designers for exploring the different tradeoffs among all objectives, and generating customized retinal models which best approximate the needs of each specific patient. Although the combination of genetic algorithm and multi-objective optimization has been widely studied, it has not been applied before for optimizing retina models. Additionally, our optimization strategy, tested and validated with a well-known retinal model derived from our previous expertise, is not bounded to any particular model. Although comparing different retinal models is beyond the scope of our paper, our framework can be considered as model-agnostic, in the sense that it is possible to simulate and optimize models from diverse nature.

The rest of the paper is structured as follows: Section 2 presents the evolutionary multiobjective strategy for tuning a retinal model; Section 3 presents briefly the multielectrode recordings from retinal ganglion cell populations; Section 4 describes and discusses the experimental cases of study, and finally, Section 5 provides the main conclusions of this work.

\section{Evolutionary multi-objective strategy for tuning a retina model}

As introduced in Section 1 considering the vast number of possible parameters which must be taken into account, the problem of automatic tuning of a retinal model cannot be achieved by exhaustively exploring all the solutions space. In addition, not one but several antagonist objectives come into play to assess the quality of a retinal model, and thus, the problem becomes multi-objective. Therefore, we propose to use a genetic algorithm for guiding the exploration of solutions and a multi-objective procedure for assessing every solution reached by the exploration. The proposed well-known evolutionary strategy is based on the assumption that evolution could be used as an optimization tool for a multi-objective problem. The idea is to evolve a population of candidate solutions using operators inspired by natural genetic variation and natural selection. In our case, the population of candidates are defined by a set of retinal models. Therefore, multi-objective optimization (MOO) based on a Genetic Algorithm (MOOGA) is adopted as strategy to explore the huge solution space. This strategy has been extensively proved by us as a suitable method of optimizing problems from diverse natures. As a matter of fact, MOOGA has been used to study and improve the reliability of embedded software when diverse hardening techniques are applied 41 , improve Apache server quality metrics when diverse compiler options are used ${ }^{42} 43$, or feature selection for network anomalies ${ }^{44}$. Furthermore, these techniques have been validated by other authors in other areas of knowledge such as integrated engineering or civil engineering 45-49, production-distribution planning problems 50 , counterrotating compressors 51, multi-label lazy algorithms 52, quantitative association rules 53 , design of in-building wireless networks 54 and others 55 59.

Genetic Algorithms (GAs) are methods for solving optimization problems. They belong to the group of techniques known as Evolutionary Algorithms (EAs) which are based on the imitation of evolutionary processes such as natural selection, crossover or mutation. Every individual in the population, which is randomly initialized, represents a possible solution 
for the problem. Thus better and better solutions evolve from previous generations until a near optimal solution is obtained.

The population is evolved through crossover and mutation operators and only those that represent better solutions are extracted from the surviving population. The selection operator chooses from the population, those individuals that will be allowed to reproduce, being the best individuals those who maximize or minimize the goals, according to their nature. In our case, several goals are considered at the same time, so a MOO strategy, (e.g. NSGA-II or SPEA2) can be used to rank the individuals. The crossover operation exchanges subparts of two individuals and recombines, imitating by this way the biological recombination. At last, mutation randomly changes the values of the alleles of the chromosome. A step-by-step algorithm to showing the dynamic of this process is presented below.

Step 1: Initialization: Generate an initial random population $P_{0}$ of $P_{S I Z E}$ individuals. Set up the number of generations $\left(N_{G E N}\right)$, mating probability $(M A T P B)$, and mutation probability $(M U T P B)$.

Step 2: Fitness assignment: Calculate fitness values of each individual in the current population

Step 3: Start the generational prccess: Current iteration $i=0$

Step 4: Mating: Perform mating process depending on the crossover probability $(M A T P B)$

Step 5: Mutating: Perform mutating process depending on the mutating probability (MUTPB)

Step 6: Evaluation: The fitness of every individual in the population is evaluated.

Step 7: Selection: Using NSGA-II or SPEA2 selection operators, a proportion of the current population is selected to breed a new generation.

Step 8: If the current iteration $i$ is less than $N_{G E N}$ continue from Step 3, otherwise continue.

Step 9: Output: Pareto fronts and final population

To solve MOO problems, many different methods of resolution have been proposed in the last couple of years. NSGA-II (Non-dominated Sorting Genetic Algorithm II) has been reported to be one of the most successful MOO algorithms 3342 and is included within of group of elitist selection algorithms that emphasize computational efficiency, such as the Strength Pareto Evolutionary Algorithms SPEA and SPEA2 ${ }^{35}$ NSGA-II algorithm establishes an order relationship among the individuals of a population mainly based on the concept of non-dominance or Pareto fronts. It is said that one solution $\mathrm{X}_{i}$ dominates other $\mathrm{X}_{j}$ if the first one is better or equal than the second in every single objective and, at least, strictly better in one of them (i.e. Pareto fronts are defined by those points in which no improvements in one objective are possible without degrading the rest of objectives). NSGA-II firstly groups individuals in a first front that contains all non-dominated individuals, that is the Pareto front. Then, a second front is built by selecting all those individuals that are nondominated in the absence of individuals of the first front. This process is repeated iteratively until all individuals are placed in some front. The crowding distance function is used to calculate the diversity of a possible solution, and its purpose is to maintain a good spread of solutions. After that, individuals of the Pareto front are sorted in descending order based on its crowding distance value. As a result, those solutions having more diversity are prioritized.

In summary, the result of the execution of the NSGA-II, is able to provide us the population sorted by non-dominated fronts and then, by the crowding distance.

\section{Chromosome codification}

In our approach, each individual represents a possible retinal model and every gene codifies the values of the parameters of the function which models the retina. As an example, Eq. (1) and Eq. (2) represents a general retinal model as described in Sec. 1. The parameters of each function will be the candidates to be encoded as genes that make up the chromosome. The variables $i, K$ and $M$ are used to identify each function which composes the weighted sum. The variables $m u(\mu), \operatorname{sigma}(\sigma)$ and $k$ are the parameters of the Gaussian filter and of the difference of Gaussians filter. 


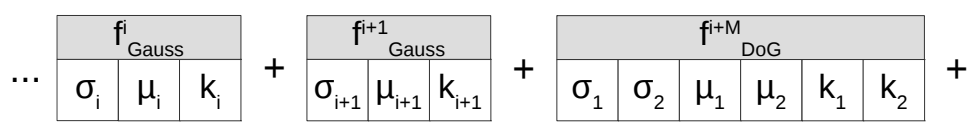

\begin{tabular}{|l|l|l|l|l|}
\hline \multicolumn{5}{|c|}{ IntegrateAndFire } \\
\hline Threshold $^{k}$ & Leakage $^{\mathrm{k}+1}$ & RefractoryPeriod $^{\mathrm{k}+2}$ & Persistence $^{\mathrm{k}+3}$ & FMF $^{\mathrm{k}+4}$ \\
\hline
\end{tabular}

Figure 2. Example of an individual codification.

$$
\begin{aligned}
\text { Stage }_{1}= & N^{i} \cdot f_{\text {Gauss }}^{i}\left(\sigma_{i}, \mu_{i}, K_{i}\right)+ \\
& N^{i+1} \cdot f_{\text {Gauss }}^{i+1}\left(\sigma_{i+1}, \mu_{i+1}, K_{i+1}\right)+\ldots \\
+ & N^{i+M} \cdot f_{D o G}^{i+M}\left(\sigma_{1}^{i+M}, \sigma_{2}^{i+M}, \mu_{1}^{i+M}, \mu_{2}^{i+M}, K_{1}^{i+M},\right. \\
& \left.K_{2}^{i+M}\right)+\ldots+N^{i+K} \cdot f^{i+K}(\ldots)+\ldots \\
\text { Stage }_{2}= & \text { IaF }(\text { Stage } 1 ; \text { CellType, Threshold } \\
& \text { Leakage, RefractoryPeriod, } \\
& \text { Persistence }) \\
& i, M, N, K \in \mathbb{N}
\end{aligned}
$$

Fig. 2 shows the way in which each gene is encoded and integrates the chromosome.

\section{Electrophysiological recordings}

To assess the MOOGA strategy and obtain a tuned retinal model, we used electrophysiological recordings from populations of mouse retinal ganglion cells. All experimental procedures were carried out in accordance with the ARVO and European Communities Council Directives (86/609/ECC) for the use of animal research.

We are trying to demonstrate the feasibility of a cortical neuroprosthesis, interfaced with the occipital cortex, as a means through which a limited but useful visual sense may be restored to profoundly blind people. One of the key challenges in this field is the design and development of a bioinspired retina-like visual processing front-end able to transform the visual world in front of a blind individual into multiple electrical signals that could be used to stimulate, in real time, the neurons at his/her visual cortex. Although visual sensors inspired by biological systems are being studied by many groups around the world, none of them is being specifically designed to make its output signal compatible with cortical neurons. In this framework we are performing multielectrode recording from isolated retinas in order to study the encoding of visual information by populations of retinal ganglion cells. Briefly, after enucleation of the eye, the eyeball was hemisected with a razor blade, and the cornea and lens were separated from the posterior half. The retinas were then carefully removed from the remaining eyecup, with the pigment epithelium, mounted on a glass slide ganglion cell up and covered with a Millipore filter. This preparation was then mounted on a recording chamber and superfused with physiological medium at 36C.

For visual stimulation, we used a 17 " high-resolution LED monitor. Pictures were focused with the help of lens onto the photoreceptor layer. The retinas were flashed periodically with full field light whereas the electrode array was lowered into the retina until a significant number of electrodes detected light evoked single- and multiunit responses. The retinas were then stimulated with moving bars and other different spatio-temporal patterns.

The electrode array was connected to a 100 channel amplifier (low and high corner frequencies of 250 and $7500 \mathrm{~Hz}$ ) and a digital signal processor based data acquisition system. All the selected channels of data as well as the state of the visual stimulus were digitized with a commercial multiplexed A/D board data acquisition system (Bionic Technologies, Inc) and stored digitally. A custom analysis program sampled the incoming data at $30 \mathrm{kHz}$, plotted the waveforms on screen, and stored single spike events for later analysis.

On the other hand, the second of block of stage 1 (see Fig. 1), is inspired in the Inner Plexiform Layer (IPL), that functions as a relay station for the vertical-information carrying nerve cells, the bipolar cells, to connect to retinal ganglion cells.

Wild-type (C57BL/6J strain) adults mice were bred within a local colony established from purchased breeding pairs (Jackson Laboratories, Bar 
Harbor, ME). Animals were dark-adapted for one hour, anesthetized with $4 \%$ of isoflurane (IsoFlo $®$, Esteve Veterinaria) and sacrificed by cervical dislocation. After enucleation of the eye, the eyeball was hemisected with a razor blade and the cornea and lens were separated from the posterior half. The retinas were then carefully removed from the remaining eye cup, mounted on a agar plate ganglion cell side up and covered with a cut Millipore filter. This preparation was then placed on a recording chamber and perfused with Ringer medium at physiological temperature. All the procedure was made under dim red illumination. Extracellular ganglion cell recordings were made using an array of 100 microelectrodes, $1.5 \mathrm{~mm}$ long (Utah Electrode Array).

Simultaneous single- and multi-unit responses were recorded with a data acquisition system (Bionic Technologies Inc) and stored on a Pentium-based computer for later analysis. Neural spike events were detected once they exceeded the thresholds established in each electrode using standard procedures described elsewhere 60]61

The spike sorting for classifying the different units was accomplished with an free open source software based on principal component analysis (PCA) method and different clustering algorithms. ${ }^{62}$ Time stamps for each action potentials of the single unit were used to generate peristimulus time histograms and peristimulus spike rasters using NeuroExplorer Version 4 (Nex Technologies) as well as customized software 63

Visual stimuli were programmed in Python using an open source library (VisionEgg) for real-time visual stimulus generation $\frac{64}{6}$ and reproduced in a 16bit ACER TFT $60 \mathrm{~Hz}$ monitor. Different patterns of light stimuli drawn on this area were projected through a beam splitter and focused onto a $4 \times 4 \mathrm{~mm}$ area photoreceptor layer with the help of optical lenses.

Finally Fig. 3 summarizes the data acquisition procedure commented bellow.

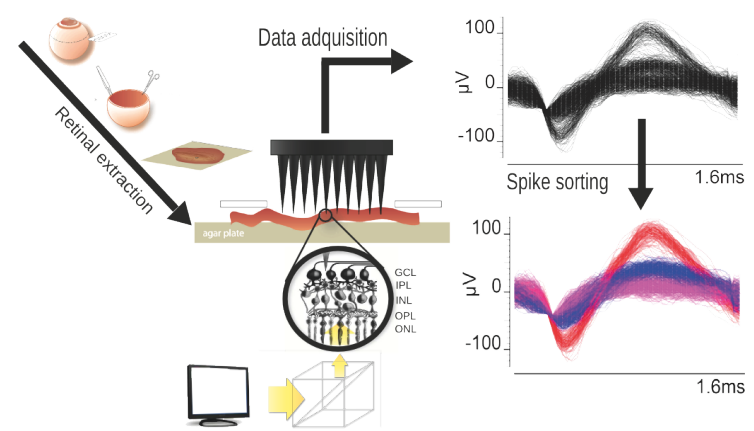

Figure 3. Data acquisition procedure.

\section{Case studies}

To assess the feasibility and quality of the proposed MOOGA strategy for tuning a retinal model, two extensive experiment-based case studies were designed. The first case study assesses the convergence of four proposed metrics, while the second one presents the multi-objective tuning of a retinal model using these metrics. In both experiments the objective was to demonstrate the relevance of a fine-tuning for a given retinal model in order to best match the electrophysiological recordings.

We choose several visual stimuli to be synthetically approximated by our retinal model. To classify the retinal ganglion cells in $\mathrm{ON}, \mathrm{OFF}, \mathrm{ON} / \mathrm{OFF}^{65}$ we used repetitions of a $700 \mathrm{~ms}$ flash $\left(196.25 \mathrm{~cd} / \mathrm{m}^{2}\right)$ followed by $2300 \mathrm{~ms}$ darkness. Then we stimulate the isolated retinas with $250 \mu \mathrm{m}$ wide white bars crossing a black screen at $0.5 \& 1 \mathrm{~Hz}$. Four pairs (eight moving bars) of stimuli were used: $0^{\circ}, 45^{\circ}, 90^{\circ}, 135^{\circ}, 180^{\circ}$, $225^{\circ}, 270^{\circ}$ and $315^{\circ}$.

The same experiments were also performed with the synthetic retinas using identical VisionEgg stimulus. Then we performed a detailed characterization of the physiological properties of the recorded neurons in both experiments (real and simulated electrophysiological recordings). To investigate the firing patterns of individual neurons we used Post-Stimulus Time Histograms (PSTH), that allow one to measure the average intensity of neuronal firing across several epochs (or bins) and raster plots.

Secondly, the selected retinal model to be tuned, which is based in the general expression already presented on Section 2, is defined on Eq. (3) and Eq. (4). 


$$
\begin{aligned}
\text { Stage }_{1}= & f_{\text {DoG }}^{1}\left(\sigma_{1}^{1} ; \sigma_{2}^{1} ; \mu_{1}^{1} ; \mu_{2}^{1} ; \kappa_{1}^{1} ; \kappa_{2}^{1}, R+B, 0.2 \cdot G\right) \\
+ & f_{\text {DoG }}^{2}\left(\sigma_{1}^{2} ; \sigma_{2}^{2} ; \mu_{1}^{2} ; \mu_{2}^{2} ; \kappa_{1}^{2} ; \kappa_{2}^{1}, R+G, B\right) \\
+ & f_{\text {LoG }}^{3}\left(\sigma_{1}^{3} ; \sigma_{2}^{3} ; \mu_{1}^{3} ; \mu_{2}^{3} ; \kappa_{1}^{3} ; \kappa_{2}^{3}, I\right) \\
\text { Stage }_{2}= & \text { IaF }\left(\text { Stage }_{1} ;\right. \text { Threshold, Leakage, } \\
& \text { RefractoryPeriod, Persistence, } \\
& \text { FrequencyModulationFactor }) ;
\end{aligned}
$$

Considering that the selected stimulus has no color (a white bar moving over a black background), we should take into account that those retinal parameters related with color processing have little or no impact on the synthetic records. Consequently the input color combination using $R, G$ and $B$ color channels, and each $\sigma$ and $\mu$ parameter from the Difference of Gaussian contribution was fixed accord-

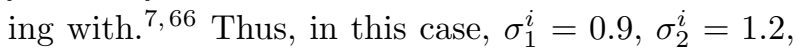
and $\mu_{j}^{i}=0$ (gaussian filters centered on each retinal ganglion cell). The $\mathrm{Stage}_{2}$ of the retinal processing model was defined by a Integrate and Fire $(\mathrm{IaF})$ spiking neuron model which was based in the LeakyIntegrate\&fFire (Leaky-IaF) model by ${ }^{67}$ with the only addition of a new parameter Persistence time to model how many times every video frame feeds the retinal model. The firing response was finally modulated by the FrequencyModulationFactor (FMF) parameter. This parameter has the effect of varying the firing pattern of a ganglion transient cell by altering its firing rate and the time to reach up to a basal response.

Each parameter from Stage $_{1}$, and the kernel size for each filter from $\mathrm{Stage}_{2}$ were selected to be automatically modified, allowing the overall tuning of the model. Regarding the kernel size parameter, each one was fixed to the same value within a given individual. I.e. each $\kappa_{j}^{i}$ from Eq. (3) remains constant for a given retinal model. Table 1 summarizes the selected parameters to be automatically changed, together with its variation range. In the case of the kernel size $K$, only odd numbers within the interval 3 to 13 are allowed. Note that, with the exception of Persistence time and $K$, the remaining parameters move within a continuous range, and thus an infinite search space is presented. The six selected parameters were codified accordingly with the chromosome scheme from Section 2

Table 1. Chromosome parameters

\begin{tabular}{lcc}
\hline Parameter & Min. value & Max. value \\
\hline K(oddnumber) & 3 & 13 \\
Threshold & 225.0 & 275.0 \\
Leakage & 10.0 & 15.0 \\
Refractory period (ms) & 1.0 & 10.0 \\
Persistence time & 3 & 7 \\
FMF & 0.25 & 0.40 \\
\hline
\end{tabular}

The parameters of the genetic operation were selected as follows: population size -60 , mutation probability -0.05 , crossover probability -0.3 and 300 iterations (or generations) for the case study presented in the Section 4.1 and 550 iterations for the case study presented in the Section 4.2 . Thus, a total amount of $60 \cdot 300=18000$ retinal models were processed in the case study 1 and $60 \cdot 550=33000$ retinal models were processed in the case study 2 . The first experiment (Section 4.1) took a total of 110 hours to be completed and the second experiment (Section 4.2 took a total of 202 hours to be completed. The simulation and calculus for each generation took 22 minutes in average using a Intel Xeon X5660 with $48 \mathrm{GiB}$ of RAM.

Finally, to compare synthetic and real electrophysiological recordings, and also to test the behavior of the proposed MOOGA strategy, four quality metrics, also called fitness functions in the field of genetic algorithms, were selected. The electrophysiological recordings coming from the experimental setup are comprised by a raster and PSTH data for each isolated ganglionar cell, which are the same type of data produced by our retina simulator. First of all, in order to compare PSTH data, KullbackLeibler Divergence (PSTH-KLD) is proposed to measure the quality of the PSTH response. This metric is widely used to compare probability distributions or histograms. A valuable and requested characteristic is its inherent sensitivity to changes on the shape of each distribution. The same metric has also been used to compare each ISI (InterSpike-interval) histogram. Secondly, to compare the area of each receptive field (RFAD), as well as the Firing Rate Absolute Difference (FRAD) of each raster recordings, we have used the absolute difference. 


\subsection{Case study 1: Study of convergence for the four proposed metrics}

Before proceeding with the multi-objective experiment, we have to test and ensure the convergence of each particular metric. To that end, four monoobjective experiments corresponding to the four presented metrics were designed to approximate both, synthetic and real electrophysiological recordings metric values. Each experiment was completed using a population of 60 individuals and a total of 300 iterations. The processing time for each mono-objective test took 22 minutes on average, and consequently it used 4.6 days of computing time. Table 2 shows the correspondence between the selected metrics and the performed experiments.

Table 2. Convergece test labeling

\begin{tabular}{ll}
\hline Quality Metric & Experiments \\
\hline$P S T H-K L D$ & Experiment 1 \\
$F R A D$ & Experiment 2 \\
$I S I-K L D$ & Experiment 3 \\
$R F A D$ & Experiment 4 \\
\hline
\end{tabular}

As can be seen from Fig. 4 all quality metrics converge at approximately the same number of iterations. Within 150 iterations the search space is reduced to $20 \%$ in each experiment, which represents fast convergence in the initial iterations. Finally, at the end of simulation, a clear convergence is obtained for each metric and thus, we validate them for the experimentation. For the sake of clarity to observe better the convergence, a magnified window using a reduced iteration margin is attached to each figure.

\subsection{Case study 2: Multi-objective tuning of retinal model}

To assess the effectiveness of the proposed MOOGA approach, a multi-objective experiment designed to find a set of retinal models optimizing at the same time a set of predefined criteria of interest is presented in this section. To this end, the four studied metrics summarized in Table 2 from Section 4.1 were selected as quality metrics in an effort to improve the retinal model presented in this section. In this experiment, we adopted the criterion of minimizing the absolute difference among synthetic and real values of each metric to approximate the electrophysiological recordings. As a result, the set of figures from Fig. 5 shows the solutions minimizing the values for all metrics at the same time. The data are graphed for pairs of criteria. Those individuals belonging to the Pareto front and consequently representing valid non-dominated solutions for the problem are identified by red dots.

Figures 5(c) to 5(f) display a collection of convex shapes for the Pareto front. On the other hand, Fig. 5(b) shows a non-convex shape of the Pareto front. In case of convex shape, it is more easily agree for a trade-off point between all possible solutions of the problem, i.e., the best retinal model.

Fig. 5(a) shows a point cloud with the set of solutions obtained comparing PSTH-KLD and FRAD metrics, which has pseudo-lineal shape. Fig. 5(b) shows the comparison between PSTH-KLD and ISI$K L D$. There are two different groups (two clusters) clearly differentiated. This is of interest as it shows that some gene is classifying the individuals of the population in two different clusters. The Pareto front in this case is composed two clusters of solutions. In Fig 5(d) FRAD and ISI-KLD fitness metrics is compared. Similary to the previous graph (Fig. 5(b) there are two different groups, more so in this case since the groups are more compact.

Fig. 5(c) Fig. 5(e) and Fig. 5(f) show similar patterns and trends, with little-differentiated clusters and little-differentiated Pareto fronts. The shape of the clusters is not defined as pronunced as on Fig. 5(b) and Fig. 5(d)

These results show that the MOOGA strategy reduces the search space and offers a set of optimized retinal models following several objectives at a time, and that the computation can be completed in a reasonable amount of time.

Finally, a visual example of how our optimization framework fits the biological behavior using the aforementioned criteria is shown in Fig. 6. This figure shows a comparison of biological and synthetic normalized PSTH and raster data produced by 9 repetitions of the experiment. Each set of raster data is represented together with the accumulated raster which gathers all repetitions for biological (up) and synthetic (bottom) cases. As usual, each PSTH is calculated using the accumulated data. In the case of synthetic data, the 9 rasters were generated by 


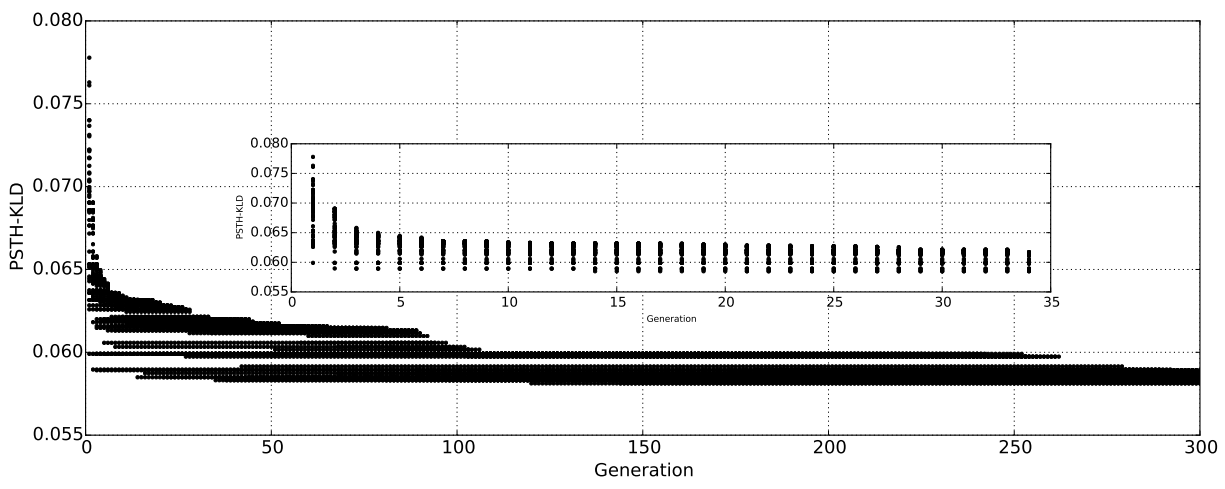

(a)PSTH-KLD convergence test (experiment 1).

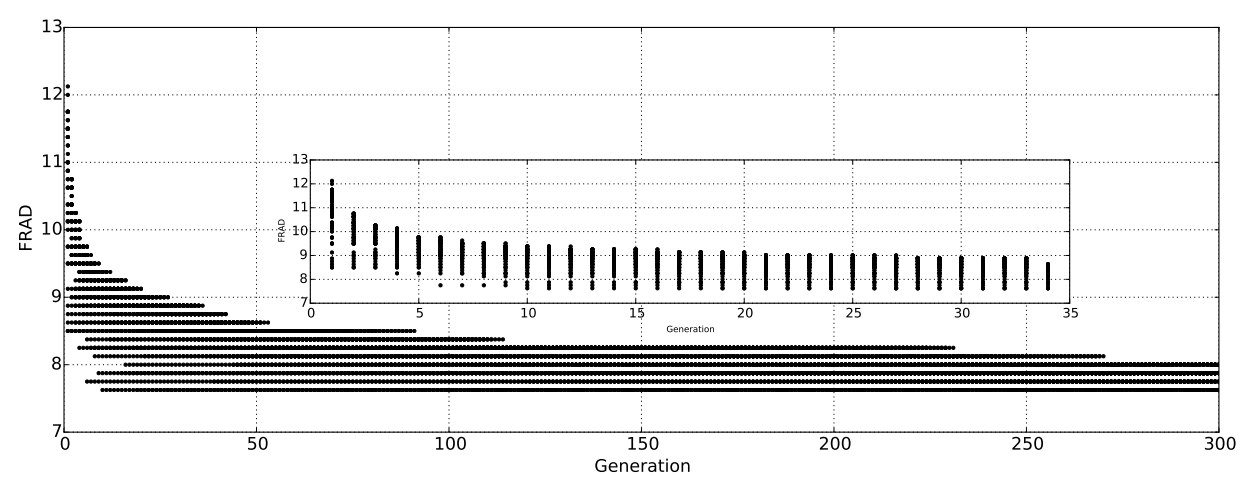

(b)FRAD convergence test (experiment 2).

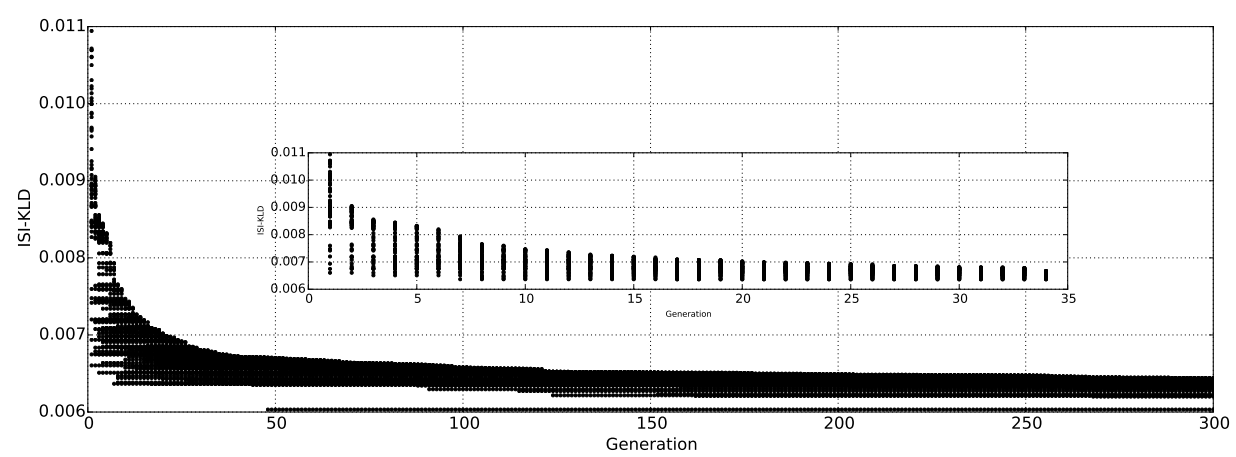

(c)ISI-KLD convergence test (experiment 3).

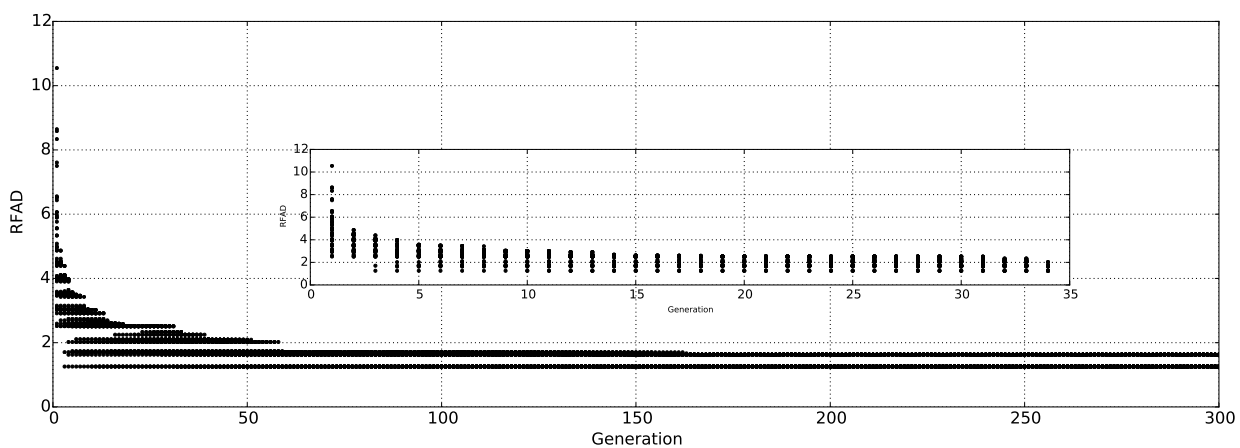

(d)RFAD convergence test (experiment 4).

Figure 4. Convergence test for PSTH-KLD (a), FRAD (b), ISI-KLD (c) and RFAD (d) when comparing biological and synthetic recordings. 


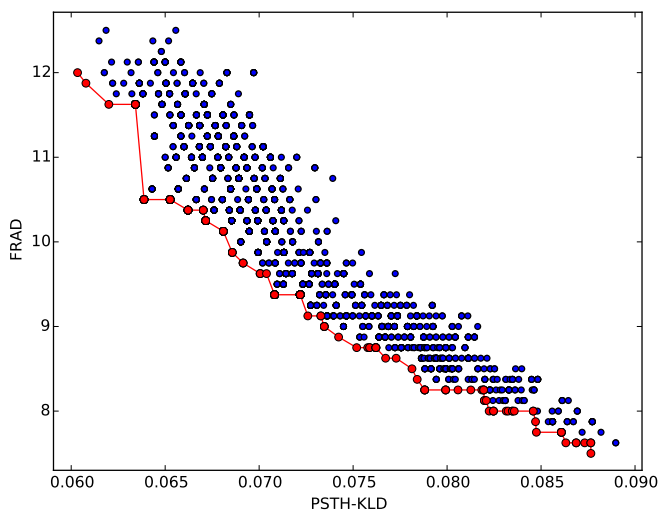

(a)PSTH-KLD against FRAD.

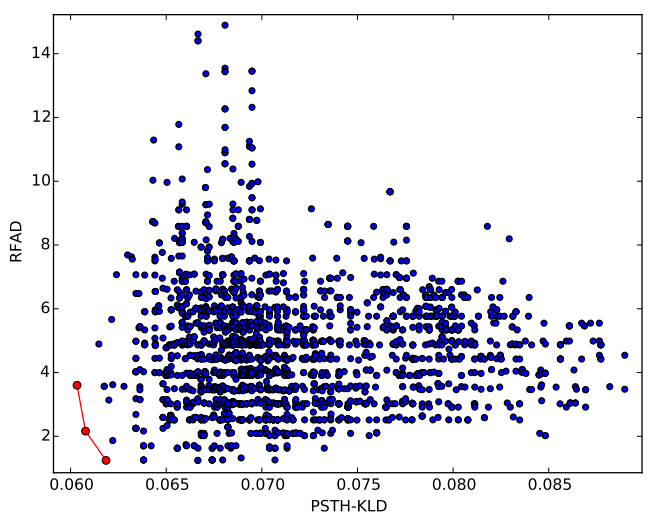

(c)PSTH-KLD against RFAD.

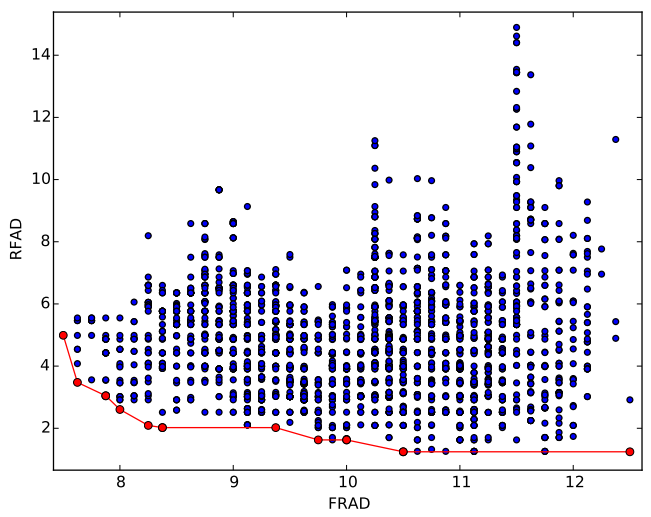

(e)FRAD against RFAD

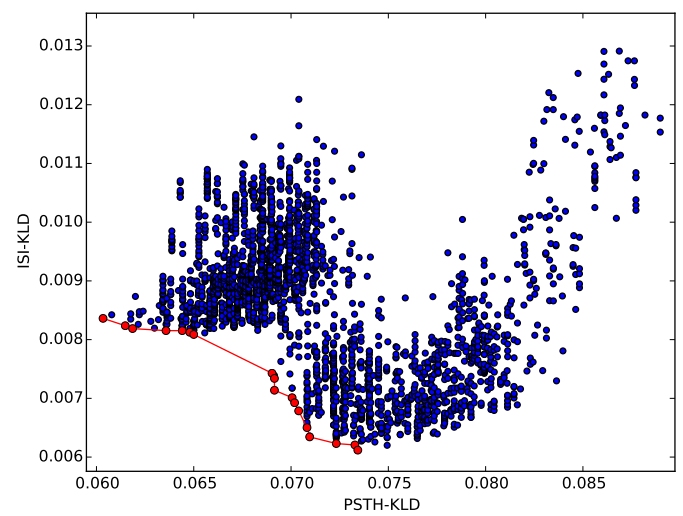

(b)PSTH-KLD against ISI-KLD.

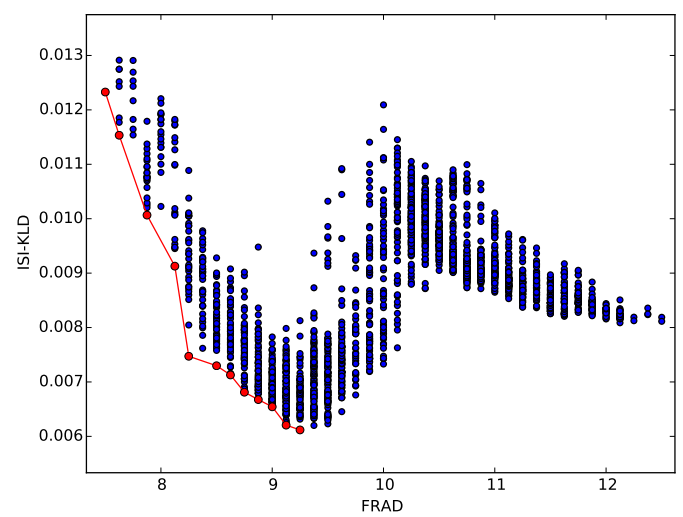

(d)FRAD against ISI-KLD.

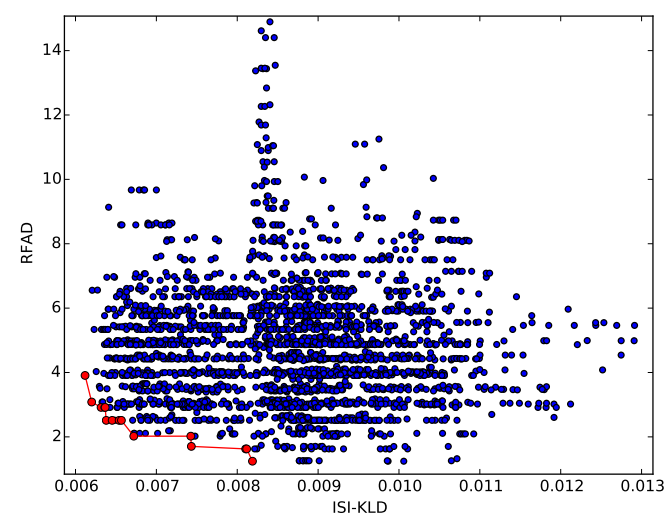

(f)ISI-KLD against RFAD.

Figure 5. Multi-objective graphs facing each pairs of criteria.

individuals selected from the Pareto front. A visual inspection of this figure reveals the tendency of ap- proximating the overall behavior of the ganglionar ON-transient cell using our MOOGA based frame- 


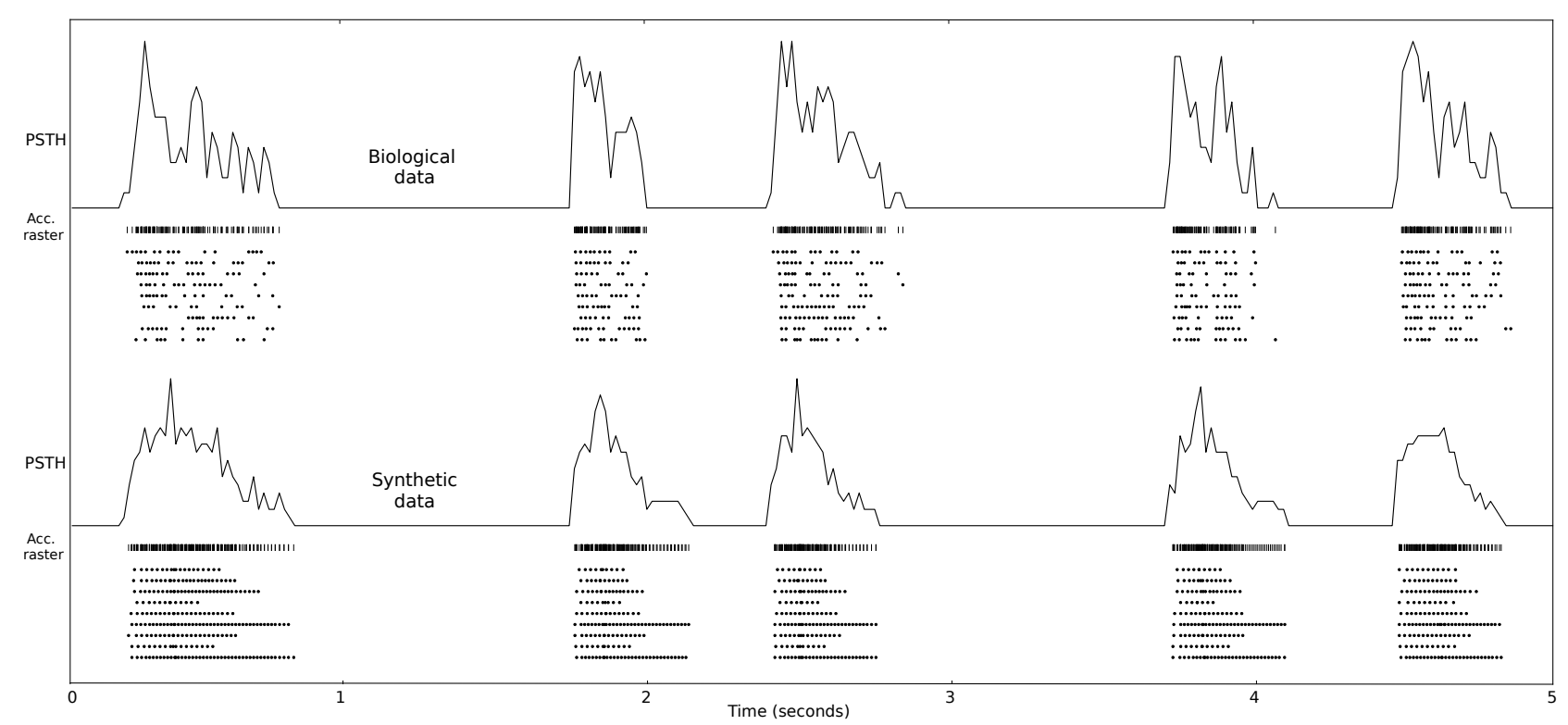

Figure 6. Comparison of biological (up) and synthetic (bottom) normalized PSTH and raster data produced by 9 repetitions of the experiment. Accumulated spikes (Acc. raster) gathering all repetitions are also shown.

\section{work.}

\section{Conclusions}

An automatic evolutionary multi-objective strategy for tuning retinal models has been presented. The tuning is performed so that a selection of those parameters that best approximate a synthetic retinal model output with actual electrophysiological recordings is automatically calculated. Our results show that this strategy performs well when dealing with optimization problems with multiple criteria satisfaction. Furthermore the selected quality metrics converge in a reasonable amount of time, what suggest that this strategy, based on the algorithm NSGA-II, is useful for adjusting neuronal models. In addition, the multi-objective strategy reveals valuable information when the proposed criteria are faced, and highlights the different trade-off among them.

\section{Bibliography}

1. S. Ghosh-Dastidar and H. Adeli, Spiking neural networks, International Journal of Neural Systems 19(4) (2009) 295-308.

2. E. Fernandez, F. Pelayo, S. Romero, M. Bongard, C. Marin, A. Alfaro and L. Merabet, Development of a Cortical Visual Neuroprosthesis for the Blind: the Relevance of Neuroplasticity, Journal of Neural Engineering 2(4) (2005) p. R1.

3. R. A. Normann, B. A. Greger, P. House, S. F. Romero, F. Pelayo and E. Fernandez, Toward the Development of a Cortically Based Visual Neuroprosthesis, Journal of Neural Engineering 6(3) (2009) p. 035001.

4. A. Ortiz-Rosario and H. Adeli, Brain-computer interface technologies: From signal to action, Reviews in the Neurosciences 24(5) (2013) 537-552.

5. C. Morillas, S. Romero, A. Martínez, F. Pelayo, L. Reyneri, M. Bongard and E. Fernández, A neuroengineering suite of computational tools for visual prostheses, Neurocomputing 70(16-18) (2007) 2817 $-2827$.

6. C. Morillas, S. Romero, A. Martinez, F. Pelayo and E. Fernández, A computational tool to test neuromorphic encoding schemes for visual neuroprostheses, Computational Intelligence and Bioinspired Systems, eds. J. Cabestany, A. Prieto and F. Sandoval, Lecture Notes in Computer Science 3512 (Springer Berlin Heidelberg, 2005), pp. 510-517.

7. A. Martínez-Álvarez, A. Olmedo-Payá, S. CuencaAsensi, J. M. Ferrández and E. Fernández, Retinastudio: A bioinspired framework to encode visual information, Neurocomputing 114(0) (2013) $45-53$.

8. J. Hérault, Vision: Images, Signals and Neural Networks (World Scientific, mar 2010).

9. A. Benoit, A. Caplier, B. Durette and J. Herault, Using human visual system modeling for bio-inspired low level image processing, Computer Vision and Image Understanding 114(7) (2010) $758-773$. 
10. M. Carandini, J. Demb, V. Mante, D. Tolhurst, Y. Dan, B. Olshausen, J. Gallant and N. Rust, Do we know what the early visual system does?, Journal of Neuroscience 25 (Nov 16 2005) 10577-10597.

11. K. Zaghloul, K. Boahen and J. Demb, Contrast adaptation in subthreshold and spiking responses of mammalian Y-type retinal ganglion cells, Journal of Neuroscience 25 (Jan 26 2005) 860-868.

12. Y. Ozuysal and S. A. Baccus, Linking the Computational Structure of Variance Adaptation to Biophysical Mechanisms, Neuron 73 (Mar 8 2012) 1002-1015.

13. J. Pillow, L. Paninski, V. Uzzell, E. Simoncelli and E. Chichilnisky, Prediction and decoding of retinal ganglion cell responses with a probabilistic spiking model, Journal of Neuroscience 25 (Nov 23 2005) 11003-11013.

14. J. W. Pillow, J. Shlens, L. Paninski, A. Sher, A. M. Litke, E. J. Chichilnisky and E. P. Simoncelli, Spatio-temporal correlations and visual signalling in a complete neuronal population, Nature 454 (Aug 21 2008) 995-U37.

15. G. Zhang, H. Rong, F. Neri and M. J. PerezJimenez, An Optimization Spiking Neural P System For Approximately Solving Combinational Optimization Problems, International Journal of $\mathrm{Neu}$ ral Systems 24 (Aug 2014).

16. S. Ghosh-Dastidar and H. Adeli, A new supervised learning algorithm for multiple spiking neural networks with application in epilepsy and seizure detection, Neural Networks 22 (DEC 2009) 1419-1431.

17. S. Ghosh-Dastidar and H. Adeli, Improved spiking neural networks for EEG classification and epilepsy and seizure detection, Integrated Computer-Aided Engineering 14(3) (2007) 187-212.

18. Z. Wang, L. Guo and M. Adjouadi, A Generalized Leaky Integrate-And-Fire Neuron Model With Fast Implementation Method, International Journal of Neural Systems 24 (Aug 2014).

19. B. Strack, K. M. Jacobs and K. J. Cios, Simulating Vertical And Horizontal Inhibition With Short-Term Dynamics In A Multi-Column Multi-Layer Model Of Neocortex, International Journal of Neural Systems 24 (Aug 2014).

20. S. Shapero, M. Zhu, J. Hasler and C. Rozell, Optimal Sparse Approximation With Integrate And Fife Neurons, International Journal of Neural Systems 24 (Aug 2014)

21. J. L. Rossello, V. Canals, A. Oliver and A. Morro, Studying The Role Of Synchronized And Chaotic Spiking Neural Ensembles In Neural Information Processing, International Journal of Neural Systems 24 (Aug 2014).

22. Q. Wu, T. M. McGinnity, L. Maguire, R. Cai and M. Chen, A visual attention model based on hierarchical spiking neural networks, Neurocomputing $\mathbf{1 1 6}$ (Sep 20 2013) 3-12, 7th International Conference on Intelligent Computing (ICIC), Zhengzhou, Peoples R China, Aug 11-14, 2011.
23. G. E. Hinton, S. Osindero and Y.-W. Teh, A fast learning algorithm for deep belief nets, Neural Computation 18 (Jul 2006) 1527-1554.

24. G. E. Hinton and R. R. Salakhutdinov, Reducing the dimensionality of data with neural networks, Science 313 (Jul 28 2006) 504-507.

25. K. Kavukcuoglu, P. Sermanet, Y. lan Boureau, K. Gregor, M. Mathieu and Y. L. Cun, Learning convolutional feature hierarchies for visual recognition, Advances in Neural Information Processing Systems 23, eds. J. Lafferty, C. Williams, J. Shawe-taylor, R. Zemel and A. Culotta 2010, pp. 1090-1098.

26. A. Krizhevsky, I. Sutskever and G. E. Hinton, Imagenet classification with deep convolutional neural networks, Advances in Neural Information Processing Systems 25, eds. P. Bartlett, F. Pereira, C. Burges, L. Bottou and K. Weinberger 2012, pp. 1106-1114.

27. D. Turcsany, A. Bargiela and T. Maul, Modelling retinal feature detection with deep belief networks in a simulated environment, 28th European Conference on Modelling and Simulation ECMS 2014. Proceedings, eds. F. Squazzoni, F. Baronio, C. Archetti and M. Castellani 2014

28. H. Lee, C. Ekanadham and A. Y. Ng, Sparse deep belief net model for visual area v2, Advances in Neural Information Processing Systems 20, eds. J. Platt, D. Koller, Y. Singer and S. Roweis 2008, pp. 873-880.

29. R. Crespo-Cano, A. Martínez-Álvarez, A. DíazTahoces, S. Cuenca-Asensi, J. Ferrández and E. Fernández, On the automatic tuning of a retina model by using a multi-objective optimization genetic algorithm, Artificial Computation in Biology and Medicine, eds. J. M. Ferrández Vicente, J. R. Álvarez Sánchez, F. de la Paz López, F. J. ToledoMoreo and H. Adeli, Lecture Notes in Computer Science 9107 (Springer International Publishing, 2015), pp. $108-118$.

30. N. Siddique and H. Adeli, Computational Intelligence: Synergies of Fuzzy Logic, Neural Networks and Evolutionary Computing (John Wiley and Sons, 2013).

31. N. Srinivas and K. Deb, Multi-objective optimization using nondominated sorting in genetic algorithms, Evol. Comput. 2 (September 1994) 221-248.

32. C. M. Fonseca and P. J. Fleming, Genetic algorithms for multiobjective optimization: Formulation, discussion and generalization, Proceedings of the 5th International Conference on Genetic Algorithms, (Morgan Kaufmann Publishers Inc., San Francisco, CA, USA, 1993), pp. 416-423.

33. K. Deb, A. Pratap, S. Agarwal and T. Meyarivan, A fast and elitist multiobjective genetic algorithm: NSGA-II, IEEE Transactions on Evolutionary Computation 6 (apr 2002) 182-197.

34. K. Deb and H. Jain, An Evolutionary ManyObjective Optimization Algorithm Using ReferencePoint-Based Nondominated Sorting Approach, Part 
I: Solving Problems With Box Constraints, IEEE Transactions on Evolutionary Computation 18 (Aug 2014) 577-601.

35. E. Zitzler, M. Laumanns and L. Thiele, SPEA2: Improving the strength pareto evolutionary algorithm for multiobjective optimization, Evolutionary Methods for Design Optimization and Control with Applications to Industrial Problems, eds. K. C. Giannakoglou, D. T. Tsahalis, J. Périaux, K. D. Papailiou and T. Fogarty (International Center for Numerical Methods in Engineering, Athens, Greece, 2001), pp. 95-100.

36. Q. Zhang and H. Li, MOEA/D: A multiobjective evolutionary algorithm based on decomposition, IEEE Transactions On Evolutionary Computation 11 (DEC 2007) 712-731.

37. E. Hughes, Multiple single objective pareto sampling, CEC: 2003 Congress On Evolutionary Computation, Vols 1-4, Proceedings, (IEEE, 2003), pp. 2678-2684.

38. J. Bader and E. Zitzler, HypE: An Algorithm for Fast Hypervolume-Based Many-Objective Optimization, Evolutionary Computation 19 (SPR 2011) 4576.

39. A. Díaz-Tahoces, A. Martínez-Álvarez, A. GarcíaMoll, L. Humphreys, J. Bolea and E. Fernández, Towards the Reconstruction of Moving Images by Populations of Retinal Ganglion Cells, Artificial Computation in Biology and Medicine, eds. J. M. Ferrández Vicente, J. R. Álvarez Sánchez, F. de la Paz López, F. J. Toledo-Moreo and H. Adeli, Lecture Notes in Computer Science 9107 (Springer International Publishing, 2015), pp. 220-227.

40. H. Adeli and S. Hung, Machine Learning: Neural Networks, Genetic Algorithms, and Fuzzy Systems (John Wiley \& Sons, 1995).

41. A. Martínez-Álvarez, F. Restrepo-Calle, L. A. V. Tejuelo and S. Cuenca-Asensi, Fault tolerant embedded systems design by multi-objective optimization, Expert Systems with Applications 40(17) (2013) 6813 $-6822$.

42. A. Martínez-Álvarez, S. Cuenca-Asensi, A. Ortiz, J. Calvo-Zaragoza and L. A. V. Tejuelo, Tuning compilations by multi-objective optimization: Application to apache web server, Applied Soft Computing 29(0) (2015) 461 - 470.

43. A. Martínez-Álvarez, J. Calvo-Zaragoza, S. CuencaAsensi, A. Ortiz and A. Jimeno-Morenilla, Multiobjective adaptive evolutionary strategy for tuning compilations, Neurocomputing 123 (2014) 381 - 389, Contains Special issue articles: Advances in Pattern Recognition Applications and Methods.

44. E. de la Hoz, E. de la Hoz, A. Ortiz, J. Ortega and A. Martínez-Álvarez, Feature selection by multi-objective optimisation: Application to network anomaly detection by hierarchical self-organising maps, Knowledge-Based Systems 71 (2014) 322 338.
45. H. Kim and H. Adeli, Discrete cost optimization of composite floors using a floating-point genetic algorithm, Engineering Optimization 33(4) (2001) 485501.

46. X. Jiang and H. Adeli, Neuro-genetic algorithm for non-linear active control of structures, International Journal for Numerical Methods in Engineering 75(7) (2008) 770-786.

47. M. Kociecki and H. Adeli, Two-phase genetic algorithm for size optimization of free-form steel spaceframe roof structures, Journal of Constructional Steel Research 90 (2013) $283-296$.

48. M. Kociecki and H. Adeli, Two-phase genetic algorithm for topology optimization of free-form steel space-frame roof structures with complex curvatures, Engineering Applications of Artificial Intelligence 32 (2014) $218-227$.

49. M. Kociecki and H. Adeli, Shape optimization of free-form steel space-frame roof structures with complex geometries using evolutionary computing, Engineering Applications of Artificial Intelligence $\mathbf{3 8}$ (2015) $168-182$.

50. L. Jia, Y. Wang and L. Fan, Multiobjective bilevel optimization for production-distribution planning problems using hybrid genetic algorithm, Integr. Comput.-Aided Eng. 21 (January 2014) 77-90.

51. M. M. Joly, T. Verstraete and G. Paniagua, Integrated multifidelity, multidisciplinary evolutionary design optimization of counterrotating compressors, Integr. Comput.-Aided Eng. 21 (July 2014) 249-261.

52. O. Reyes, C. Morell and S. Ventura, Evolutionary feature weighting to improve the performance of multi-label lazy algorithms, Integr. Comput.-Aided Eng. 21 (October 2014) 339-354.

53. J. M. Luna, J. R. Romero, C. Romero and S. Ventura, Reducing gaps in quantitative association rules: A genetic programming free-parameter algorithm, Integrated Computer-Aided Engineering 21 (29 September 2014) 321-337.

54. M. Molina-García, J. Calle-Sánchez, C. GonzálezMerino, A. Fernández-Durán and J. I. Alonso, Design of in-building wireless networks deployments using evolutionary algorithms, Integr. Comput.-Aided Eng. 21 (October 2014) 367-385.

55. P. C. D. Paris, E. C. Pedrino and M. C. Nicoletti, Automatic learning of image filters using cartesian genetic programming, Integr. Comput.-Aided Eng. 22 (April 2015) 135-151.

56. L. F. S. Coletta, E. R. Hruschka, A. Acharya and J. Ghosh, Using metaheuristics to optimize the combination of classifier and cluster ensembles, Integrated Computer-Aided Engineering 22(3) (2015) 229-242.

57. D.-Y. Lin and Y.-H. Ku, Using genetic algorithms to optimize stopping patterns for passenger rail transportation, Computer-Aided Civil and Infrastructure Engineering 29(4) (2014) 264-278.

58. W. Zhu, H. Hu and Z. Huang, Calibrating rail transit 
assignment models with genetic algorithm and automated fare collection data, Computer-Aided Civil and Infrastructure Engineering 29(7) (2014) 518530.

59. H.-G. Lee, C.-Y. Yi, D.-E. Lee and D. Arditi, An advanced stochastic time-cost tradeoff analysis based on a cpm-guided genetic algorithm, Computer-Aided Civil and Infrastructure Engineering 30(10) (2015) 824-842.

60. E. Fernández, J.-M. Ferrández, J. Ammermüller and R. A. Normann, Population coding in spike trains of simultaneously recorded retinal ganglion cells1, Brain Research 887(1) (2000) 222 - 229.

61. R. A. Normann, D. J. Warren, J. Ammermuller, E. Fernandez and S. Guillory, High-resolution spatiotemporal mapping of visual pathways using multielectrode arrays, Vision Research 41(10-11) (2001) $1261-1275$.

62. M. Bongard, D. Micol and E. Fernández, Nev2lkit: A new open source tool for handling neural event files from multi-electrode recordings, International Journal of Neural Systems 24(04) (2014) p. 1450009,
PMID: 24694167.

63. G. J. Ortega, M. Bongard, E. Louis and E. Fernández, Conditioned spikes: a simple and fast method to represent rates and temporal patterns in multielectrode recordings, Journal of Neuroscience Methods 133(1-2) (2004) 135 - 141.

64. A. D. Straw, Vision egg: an open-source library for realtime visual stimulus generation, Frontiers in Neuroinformatics 2(4) (2008).

65. M. Van Wyk, H. Wässle and W. R. Taylor, Receptive field properties of on- and off-ganglion cells in the mouse retina, Visual Neuroscience 26 (5 2009) 297-308.

66. F. J. Pelayo, S. Romero, C. A. Morillas, A. Martínez, E. Ros and E. Fernández, Translating image sequences into spike patterns for cortical neurostimulation, Neurocomputing 58-60(0) (2004) 885892.

67. W. Gerstner and W. M. Kistler, Spiking Neuron Models: Single Neurons, Populations, Plasticity (Cambridge University Press, 2002). 